Previous | Up | Next Article

MR2140050 (2006c:12010a) 12H05 (03C60)

Kirby, Jonathan (4-OX)

A Schanuel condition for Weierstrass equations. (English summary)

J. Symbolic Logic 70 (2005), no. 2, 631-638.

Citations

From References: 0

From Reviews: 0

MR2158459 (2006c:12010b) 12H05 (03C60)

Kirby, Jonathan (4-OX)

Corrigendum to: "A Schanuel condition for Weierstrass equations" [J. Symbolic Logic 70 (2005), no. 2, 631-638; MR2140050 (2006c:12010a)].

J. Symbolic Logic 70 (2005), no. 3, 1023.

Consider an ordinary differential field $F$ of characteristic zero with differentiation $\delta$ and $C$ as the field of constants. For $n$ solutions in $F$ of the equation $\delta y_{i}=y_{i} \delta x_{i}$ we have tr. $\operatorname{deg} \cdot{ }_{C} C\left(x_{1}, y_{1}, \ldots, x_{n}, y_{n}\right) \geq n+1$ if the elements $\delta x_{i}$ are linearly independent over $C$ [see J. Ax, Ann. of Math. (2) 93 (1971), 252-268; MR0277482 (43 \#3215)]. The paper under review studies such a problem for another differential equation, namely, for the Weierstrass one: $(\delta y)^{2}=$ $f(y)(\delta x)^{2}$, where $f$ is a cubic with constant coefficients and without multiple roots.

The author first gives a similar result with one equation and many solutions (Proposition 1.1), and then does this having several different cubics $f_{i}$ (Proposition 1.2) with a certain extra condition for relations between $f_{i}$. Finally, these results are generalized for a partial differential field $F$ with the basic set of differentiations $\Delta=\left\{\delta_{1}, \ldots, \delta_{s}\right\}$. The statement is similar: tr. deg ${ }_{C} C\left(\left\{x_{i k}, y_{i k}\right\}\right) \geq$ $\sum_{i} n_{i}+r$, where $r$ is the rank of the Jacobi matrix $\left(\delta_{l} x_{i k}\right)$ and $\left(x_{i k}, y_{i k}\right)$ are $n_{i}$ solutions for the corresponding Weierstrass equations with different cubics $f_{i}$.

In the corrigendum the author provides corrections for the two statements of the paper under review: the conditions for the relations between cubics $f_{i}$ in Propositions 1.2 and 3.2 should be given in a stronger way.

Reviewed by Alexey I. Ovchinnikov

\title{
References for 2006c:12010a
}

1. James Ax, On Schanuel's conjectures, Annals of Mathematics, vol. 93 (1971), pp. 252-268. MR0277482 (43 \#3215)

2. Cristiana Bertolin, P'eriodes de 1-motifs et transcendance, Journal of Number Theory, vol. 97 (2002), pp. 204-221. MR1942957 (2003i:11104)

3. W. Dale Brownawell and K. K. Kubota, The algebraic independence of Weierstrass functions and some related numbers, Acta Arithmetica, vol. XXXIII (1977), pp. 111-149. MR0444582 (56 \#2932)

4. Ehud Hrushovski, A new strongly minimal set, Annals of Pure and Applied Logic, vol. 63 (1993), no. 2, pp. 147-166. MR1226304 (94d:03064) 
5. E. R. Kolchin, Galois theory of differential fields, American Journal of Mathematics, vol. 75 (1953), pp. 753-824. MR0058591 (15,394a)

6. David Marker, Model theory of differential fields, Model Theory of Fields (David Marker, Margit Messmer, and Anand Pillay, editors), Lecture Notes in Logic, vol. 5, Springer, 1996.

7. A. Seidenberg, Abstract differential algebra and the analytic case, Proceedings of the American Mathematical Society, vol. 9 (1958), no. 1, pp. 159-164. MR0093655 (20 \#178)

8. , Abstract differential algebra and the analytic case II, Proceedings of the American Mathematical Society, vol. 23 (1969), no. 3, pp. 689-691. MR0248122 (40 \#1376)

9. Joseph Silverman, The Arithmetic of Elliptic Curves, Graduate Texts in Mathematics, vol. 106, Springer, 1986. MR0817210 (87g:11070)

10. Boris Zilber, Complex geometry and pseudo-analytic structures, Oberwolfach tutorial, available on http://www.maths.ox.ac.uk/ zilber, 2004.

11. , Analytic and pseudo-analytic structures, Logic colloquium 2000 (R. Cori et al., editors), Lecture Notes in Logic, vol. 19, AK Peters, 2005, pp. 392- 408. MR2143889 (2006d:03058)

Note: This list, extracted from the PDF form of the original paper, may contain data conversion errors, almost all limited to the mathematical expressions.

\section{References for 2006c:12010b}

1. Jonathan Kirby, A Schanuel condition for Weierstrass equations, this Journal, vol. 70 (2005), no. 3, pp. 631-638. MR2140050 (2006c:12010a)

Note: This list, extracted from the PDF form of the original paper, may contain data conversion errors, almost all limited to the mathematical expressions.

(c) Copyright American Mathematical Society 2006, 2011 